PROFIL VERBINDUNGSTECHNIK

RND-LIGHT-NIETMUTTER

\section{ZUR GEWICHTSREDUZIERUNG}

Gegenüber den gebräuchlichen Nietmuttern lassen sich mit der RND light der Friedrichsdorfer Profil Verbindungstechnik bis zu 50 \% Gewicht einsparen, ohne Einbußen bei den bekannt hohen Festigkeitseigenschaften im Blech hinnehmen zu müssen. Die runde Nietmutter für hohe dynamische Belastungen kann in einem breiten Bereich von 0,6 mm bis 4,0 mm Blechdicke verwendet werden. Auch werkstoffseitig ist die RND light laut Profil Verbindungstechnik vielseitig einsetzbar. Das Anwendungsspektrum reiche von Stahl, hochfesten Stählen und Edelstählen über NE-Metalle, wie Aluminium, bis hin zu oberflächenbeschichteten Blechen.

Die RND light wird in vorgelochte Blechteile eingenietet. Dabei wird das Blech formteil zwischen d e m Flansch der Mutter und dem Nietabschnitt sowohl kraft- als auch formschlüssig befestigt. „Somit entsteht eine in Zug- und Druckrichtung dynamisch und statisch hochbelastbare Verbindung, ohne das Blechformteil zu schwächen", heißt es.

Weitere Vorteile sind laut Unternehmen hohe Ausknöpfmomente, hohe Verdreh- und Durchzugswiderstände, keine Gefügeveränderungen im Blechformteil und keine Funktionsbeeinträchtigung durch Schweißspritzer, wie sie bei termisch gefügten Verbindungselementen entstehen. Erste Serienfreigaben in der Automobilindustrie lägen bereits vor.

Als Systemanbieter liefert Profil außer den Verbindungselementen auch die dazu gehörende Verarbeitungstechnik. Neben standardisierten Anlagen umfasse das Portfolio auch maßgeschneiderte Systemlösungen für optimale Wirtschaftlichkeit. Zur „Alles-aus-einer-Hand“-Philosophie gehöre eine umfassende technische Beratung, eigene Entwicklung, eine hohe Fertigungstiefe und ein vor Ort-Service mit kurzer Reaktionszeit.

\title{
ZWICK
}

\section{Torsionsprüfung mit hoher Auflösung und Wiederholgenauigkeit}

Torsionsprüfmaschinen werden in fast alIen Branchen der Industrie genutzt, um Drehwinkelmessungen über einen definierten Drehmomentbereich an Werkstoffen und Bauteilen durchzuführen. Zentrale Parameter sind Drehmoment, Auflösung und Wiederholgenauigkeit. Für die unterschiedlichen Anforderungen, auch in Bezug auf die Aufstellungsmöglichkeiten, bietet Zwick verschiedene Prüfmaschinen an: TorsionLine, ZwickiTorsion sowie Tisch-/ Standprüfmaschinen mit Torsionsantrieb.

"Alle Torsionslösungen bauen auf Zwick Standard-Lastrahmen auf. Das heißt, sie bieten dem Anwender eine hohe Flexibilität, da die Maschinen mit allen verfügbaren Standard-Werkzeugen und Software-Modulen genutzt werden können", teilt das Ulmer Unternehmen mit. Hinzu komme ein umfangreiches optionales Zwick-Zubehörprogramm.

Die TorsionLine mit wartungsfreien ACServo-Antrieben und horizontaler Prüfachse ist für 20, 200, 500, 1.000 und $2.000 \mathrm{Nm}$ konzipiert. Sie biete ein Höchstmaß an Steifigkeit und gewähr- leiste eine sehr präzise Drehwinkelmessung über den gesamten Drehmomentbereich. Aufgrund der hohen Auflösung werde darüber hinaus eine hohe Wiederholgenauigkeit erreicht.

Zur ein- und zweiachsigen Torsionsprüfung (Zug-oder Druck kombiniert mit Torsion) in der Werkstoff- und Bauteilprü-

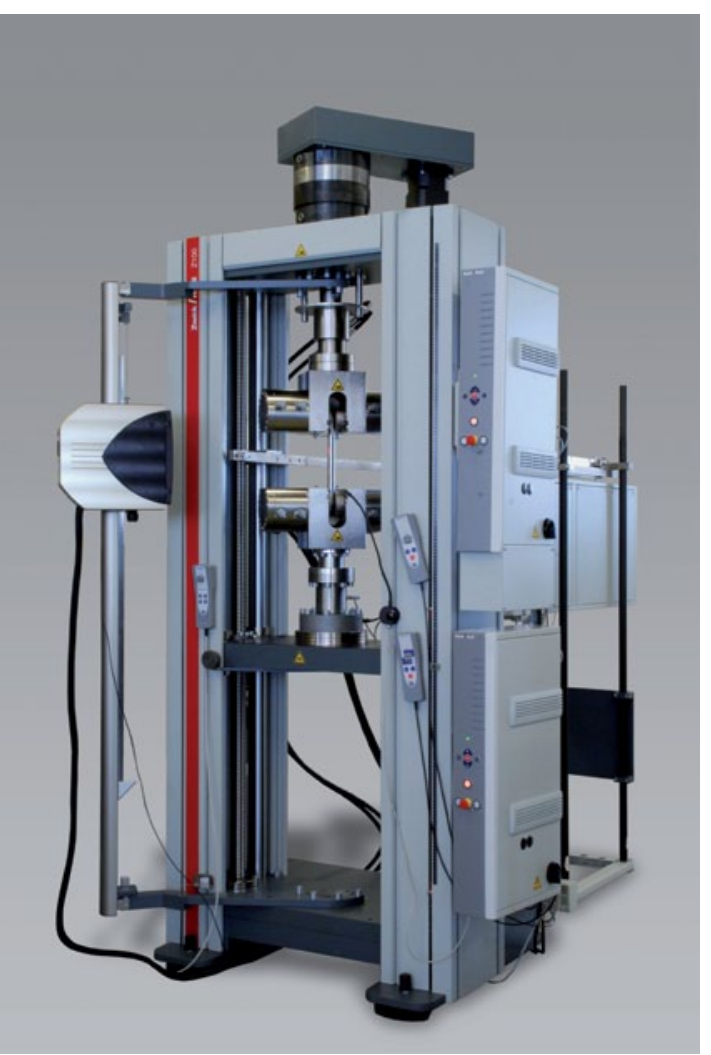

fung gebe es die Torsionsantriebe auch in Verbindung mit "zwickiLine-Materialprüfmaschinen". Diese Kombination, bei der beide Prüfachsen über die Software synchronisiert werden können, ermögliche Prüfungen von 2 bis $20 \mathrm{Nm}$. "Eine weitere Lösung ist die Integration von Torsionsantrieben in Tisch- und Standprüfmaschinen für Drehmomente von 2 bis $200 \mathrm{Nm}$, beziehungsweise 100 bis 2.000 Nm", heißt es weiter. Der Torsionsantrieb könne sowohl auf der Kopftraverse als auch auf der Fahrtraverse angebaut werden. Bei Bedarf könnten die Drehmomentaufnehmer auch mit einem Kraftaufnehmer kombiniert werden. Bei allen genannten Maschinen werde der Anwender bei der Torsionsprüfung von der Mess-, Steuer- und Regelelektronik testControl unterstützt. 